\title{
Fundamental Laws of Nature and Picture of the World
}

\author{
Vyacheslav Mikhailovich Somsikov' ${ }^{1}$, Svetlana Nikolaevna Azarenko² \\ ${ }^{1}$ Al-Farabi Kazakh National University, Almaty, Kazakhstan \\ ${ }^{2}$ Almaty Academy of the Ministry of Internal Affairs of the Republic of Kazakhstan, Almaty, Kazakhstan \\ Email:vmsoms@rambler.ru,sveta.azarenko@gmail.com
}

How to cite this paper: Somsikov, V. M., \& Azarenko, S. N. (2021). Fundamental Laws of Nature and Picture of the World. Open Journal of Philosophy, 11, 292-306. https://doi.org/10.4236/ojpp.2021.112020

Received: March 18, 2021

Accepted: May 9, 2021

Published: May 12, 2021

Copyright $\odot 2021$ by author(s) and Scientific Research Publishing Inc. This work is licensed under the Creative Commons Attribution International License (CC BY 4.0).

http://creativecommons.org/licenses/by/4.0/

\section{(c) (i) Open Access}

\begin{abstract}
The question of constructing an evolutionary picture of the world based on the results obtained by extending classical mechanics is considered. The expansion of mechanics arose as a result of taking into account the role of the structure of bodies in their dynamics. It is shown that such an extension leads to the possibility of combining branches of physics, in particular, to the substantiation of the laws of thermodynamics, statistical physics, kinetics within the framework of the laws of classical mechanics. It turned out that, according to the laws of classical mechanics, matter is infinitely divisible and can be represented by an infinite hierarchical structure from simple to complex. The expansion showed the existence of universal principles connecting the laws of the upper rung of the hierarchical ladder of matter with the laws of the lower rung. It is considered how they lead to the possibility of constructing a picture of the world based on the fundamental laws of nature.
\end{abstract}

\section{Keywords}

Determinism, Evolution, The Word's Picture

\section{Introduction}

The creation of an evolutionary physical picture of the world is the main goal of physics and science in general. There are many challenges to achieving this goal. One of them refers to the problems of epistemology, because science today consists of a large number of areas that are weakly interconnected. This is largely due to the variety of forms of matter and their behavior, in accordance with which these directions were created. For example, classical mechanics describes the dynamics of systems in space based on fundamental laws of physics, and thermodynamics describes the internal properties of systems based on pheno- 
menological laws. Moreover, the second law of thermodynamics, which is common to all natural science, which studies both living and bone matter, does not agree with the laws of classical mechanics. Statistical physics aims to substantiate the laws of thermodynamics based on statistical laws for systems. These laws have a probabilistic nature that does not correspond to the determinism of classical mechanics. Quantum mechanics studies the physics of elementary particles, the laws and principles of which do not agree well with the deterministic laws of classical mechanics. That is, the creation of a unified picture of the world is faced with seemingly insurmountable obstacles due to the contradictions of theories in various fields of science, the specifics of research in each area of knowledge, insufficiently strong positions of determinism. It is obvious that the solution of these problems will be greatly simplified if the fundamental laws of nature are discovered, the consequence of which would be not only all branches of physics, but also the laws that determine the functioning of living organisms. Fundamental laws should include such laws of physics "which cannot be explained using deeper principles", or such laws that unite all known forces (Weinberg, 1922; Hooft, 2017). Leaving aside the important question of the differences between these two types of laws, we note only that they are united by the idea of the possibility of constructing on their basis an evolutionary physical picture of the world. The necessity of the existence of such laws follows from the conditions of the unity of the world and the cognizability of natural phenomena. This also follows from the principles of causality and sufficient reason, as well as the evolutionary origin of forms of matter from simple to complex (Baumgarten, 2017). It is also important for us that only based on fundamental physical laws it is possible to build a closed and self-consistent scientific picture of the world.

But even with the fundamental laws of nature, we will still face great difficulties. Indeed, the huge variety of forms of matter makes it almost impossible to study each of these forms, if there is the same huge variety of approaches to their study. However, a way out of this situation is not excluded, which may be dictated by nature itself. Indeed, if we proceed from the ideas of the evolutionary origin of matter, then this matter should be an endless hierarchical ladder that rises from the simple to the complex (Somsikov, 2021). Then, given the infinite variety of forms of matter, these difficulties are surmountable if there are universal principles of transition between the steps of the hierarchical ladder of matter. That is, if there are universal principles, then knowledge of the laws of behavior at the lower hierarchical levels of matter, allows you to determine the behavior of matter at the upper hierarchical levels. Based on these principles, it is possible to build a hierarchical picture of the world according to a universal scheme, starting from the knowledge of the laws of nature for any of the steps of the hierarchical ladder of matter.

Thus, in order to create an evolutionary picture of the world, it is necessary to solve two key questions. First, are there fundamental laws of nature, from which the evolutionary physical picture of the world follows consistently and unambiguously? Secondly, are there universal principles, the knowledge of which, re- 
gardless of the forms of matter, allows, from the knowledge of the laws of behavior of matter at the lower rungs of the hierarchical ladder of matter, to rise to the knowledge of laws at the upper rungs? The solution of these questions is an independent big problem of our time, but here we restrict ourselves to considering only how the physics of evolution, which arose based on an extension of classical mechanics, answers them. The task of the physics of evolution includes the description of the picture of the world based on the fundamental laws of nature (Somsikov, 2019).

\section{Expansion of Classical Mechanics and Construction of an Evolutionary Picture of the World}

Any scientific theory at the first stages is built within the framework of knowledge that exists at the time of the creation of these theories, based on models, hypotheses, axioms (Baumgarten, 2017). Therefore, theories are always an approximate reflection of reality. In the process of developing knowledge, theories inevitably encounter problems of explaining new natural phenomena. Consequently, there is a need for the constant development of theories over time. As a rule, this can be done by removing the restrictions previously adopted in them. Such a need arose in classical mechanics as well. This was mainly caused by the contradiction between the reversibility of Newton's equation of motion with the canonical formalism of classical mechanics and the irreversibility of the observed natural phenomena (Zaslavsky, 1984). This contradiction makes it impossible to describe evolutionary processes within the framework of the basic laws of physics.

When constructing mechanics, as a model of a body, Newton used its simplest model in the form of a Material Point (MP), which has no structure and dimensions. Despite the significant simplification of the model of bodies, this made it possible to determine the basic laws of the dynamics of matter. This marked the beginning of the development of modern physics and opened up the possibility of solving a huge number of problems and tasks at that time. But with the development of knowledge, classical mechanics and its formalisms encountered serious difficulties in describing the observed phenomena of nature.

Experiments have shown that, in accordance with Newton's second law, the acceleration of bodies is always proportional to the applied sum of forces. But the work of active forces is always less than it should be in accordance with the work expended on their movement. Moreover, there is a maximum speed of a body, at which its acceleration is equal to zero for a given force. Already in the process of constructing the theory of classical mechanics, it became clear that this is due to the forces of friction. At first, all the difficulties arising associated with the need to take into account friction were rather easily eliminated with the help of empirical terms introduced into the right-hand side of Newton's equation of motion. And although the nature of the friction forces remained unclear, this fact was not given due attention, since the agreement with the experiments was not bad. But over time, it became clear that a lack of understanding of the 
forces of friction leads to serious problems. These, in particular, include the problems of describing the processes of the emergence of structures of matter, the problem of establishing equilibrium in closed systems. Attempts to solve them, relying on the already known formalisms of classical mechanics, have not been crowned with success.

Boltzmann was the first to find that to understand these problems; first, it is necessary to solve the problem of irreversibility. However, the solution he proposed later required the use of probabilistic principles that are alien to the deterministic laws of classical mechanics (Zaslavsky, 1984). This excluded the possibility of constructing a deterministic evolutionary picture of the world, since the probabilistic principles and fundamental laws of physics are incompatible (Landau \& Lifshits, 1976). The problem was removed only after the problem of taking into account the role of the structure of bodies in their dynamics was formulated and solved. As a result of its solution, an equation of body motion was constructed, in which, instead of its model in the form of an MP, the model of a structured body (SB) was used (Somsikov, 2021). As in statistical physics, an equilibrium system of potentially interacting MP was used as a SB (Landau \& Lifshits, 1976). Thanks to this, the concept of entropy was included in the description of the dynamics of systems. Later, based on the SB equation of motion, it was shown that structure is a necessary property of matter. This follows from the very laws of mechanics and the principle of its evolutionary origin (Somsikov, 2007).

Thus, taking into account the structure of bodies already in the very equation of motion led not only to the expansion of the framework of classical mechanics and its formalisms, but also opened the possibility of constructing an evolutionary picture of the world. To clarify these statements, let us show what extension of classical mechanics arises because of taking into account the role of body structures in their dynamics and how it was obtained.

Today it is known that the energy of motion of a body, its moment and momentum, the very equation of motion are determined by the symmetries of space and time (Landau \& Lifshits, 1973; Mac Voy, 1967). In particular, from the condition of homogeneity of time, the law of conservation of energy follows, according to which the energy of motion of a body is conserved along its trajectory. The equation of motion of the MP follows directly from the law of conservation of energy of motion (Lanczos, 1965). But if the body has a structure, then in addition to the energy of motion, it also contains internal energy. On the other hand, everyday experience shows that the energy of a body's motion can be spent on heating it, that is, on increasing the body's entropy because of friction. Hence, it is clear that the work expended on the acceleration of the body must always be less than it follows from Newton's equation of motion. This is due to the transformation of some part of the energy of motion into internal energy because of the work of friction forces arising from the movement of bodies. That is, the law of conservation of energy of motion, which follows for MP from the condition of homogeneity of time, is usually not observed for SB. This means 
that the work of friction forces leads to a violation of the temporal symmetry. But the evolution of systems is always associated with the violation of the symmetry of time. That is, the construction of an evolutionary picture of the world based on the MP equation of motion, that is, without taking into account the symmetry of bodies, is impossible. As already noted, this can also be seen from statistical physics, which is built based on representing systems as a set of equilibrium subsystems. This also follows from numerous unsuccessful attempts to find the irreversibility mechanism within the framework of Hamilton's formalism without using the hypothesis of the presence of random fluctuations. Thus, although the use of probabilistic laws greatly simplifies the analysis of systems, they are unacceptable for explaining the physics of evolution (Landau \& Lifshits, 1973). Indeed, the use of probabilistic laws to describe evolutionary processes excludes the possibility of determining the laws of formation of the vector of the evolution of matter.

Internal energy is determined by the symmetry of the body structure. The energy of motion is determined by the symmetry of space. Therefore, to describe the dynamics of bodies taking into account the effect of the structure on it, in addition to taking into account the symmetry of space, as is done in mechanics for a structureless MP, it is also necessary to take into account the symmetry of the SB. That is, the dynamics of SBs is determined by both the symmetry of the space in which they move and the internal symmetry of the SBs themselves. This has been called the principle of symmetry dualism in mechanics (Somsikov, 2010). That is, in order to take into account the dissipative processes of transforming the energy of motion into internal energy when describing the dynamics of SB, it is necessary to rely on the principle of symmetry dualism. But for this it is necessary to construct such formalisms of mechanics that take into account the interrelation of symmetries. This relationship is carried out by nonlinear terms of the equations of motion of bodies, which determine the "engagement" of independent variables belonging to two types of symmetries-symmetries of bodies and symmetries of space. In accordance with this, formalisms for SB mechanics were built based on the principle of symmetry dualism by representing energy in macro- and micro-variables that determine both the SB dynamics in space and the internal dynamics of SB elements. Moreover, micro-variables determine the internal dynamics of the MP, and the macro-variables determine the dynamics of the MP as a whole. As it turned out, the entanglement of these variables leads to irreversibility (Somsikov, 2010).

As follows from the mechanics of SB, the independence of the variables of the two symmetry groups, the invariance of the energy of motion and the reversibility are fulfilled only in the absence of friction forces. That is, according to the principle of symmetry dualism, the invariant of motion is not the energy of motion of the system, as in the case of the motion of one MP, but the sum of the energy of motion and internal energy. This obviously follows from the additivity of the energies of all MP systems and the possibility of representing the systems by a set of potentially interacting MPs (Zaslavsky, 1984). 
Let us make an important note. The fact that Newton's equation of motion follows only from the symmetry of space, and the fact that it allows one to describe the dynamics of bodies without taking into account their structure, is explained by the quadratic smallness of the dissipative terms corresponding to terms with "binding" variables.

Taking into account the structure of bodies in their dynamics based on the principle of symmetry dualism made it possible to explain the mechanisms of violation and irreversibility of temporal symmetry within the framework of the deterministic laws of physics. According to the equation of motion of the SB, irreversibility is due to the transformation of the energy of motion of the SB into its internal energy due to the work of friction forces during the movement of the body in an inhomogeneous field of forces. Note that the energy of motion can also be converted into the energy of rotation of the body, which is also a violation of translational symmetry.

As it turned out, the nature of non-potential friction forces is associated with gradients of external potential forces or, which is equivalent, with the difference in forces acting on the elements of the body. Thus, the evolution of matter is determined based on the principle of symmetry dualism and arises when it moves in an inhomogeneous space.

The explanation of the irreversibility and nature of the violation of the symmetry of time, in turn, removed the main contradictions of physics: the reversibility of the MP motion and the irreversibility of the SB motion. Earlier, attempts were made to eliminate this contradiction using the hypothesis of the existence of arbitrarily small external fluctuations (Zaslavsky, 1984). But, as it turned out, this contradiction can be eliminated within the framework of the deterministic laws of physics, without resorting to the concept of the randomness of external influences on the basis of the principle of symmetry dualism. It was this that opened the way to the construction of the physics of evolution, that is, to the description of the processes of origin, development and decay of systems based on the fundamental laws of physics (Somsikov, 2019; Somsikov, 2021).

Thus, the deterministic mechanism of irreversibility connects the laws of mechanics of MPs with the laws of mechanics of systems consisting of potentially interacting MPs. This means the determinism of the law of transition "quantity into quality" for physics and removes the main argument of agnostics about the irreducibility of knowledge of the whole to knowledge of its parts. Indeed, Newton's equation of motion for MP is reversible. And the equation of motion of a body, which is a system of potentially interacting MPs, has a qualitatively new property-irreversibility. Moreover, Newton's equation follows from the equation of motion of the SB, if friction is neglected.

It turned out that the loss of the possibility of describing the processes of evolution within the framework of the formalisms of classical mechanics was caused by the use in their conclusions of the hypothesis about the potentiality of collective forces in the system. The hypothesis is based on the well-established belief 
that if MP interactions are potential, then all collective forces of interaction between their systems will also be potential (Goldstein, 1975; Lanczos, 1965). In fact, the use of this hypothesis would be legitimate if all the collective external forces acting on the system were determined only by their summation. But, as already noted, non-potential forces arise in moving systems in an inhomogeneous force field. They are determined not by the addition of external forces acting on the elements of the system, but by their gradients (Somsikov, 2016; Somsikov, 2020). In a uniform force field, micro- and macro-variables do not interact, the internal energy SB does not change, and Newton's equation is valid.

\section{Energy and Entropy, "Order" and "Chaos"}

The concepts of energy and entropy play a key role in all areas of natural science. These, in a sense, opposite, but complementary concepts can be associated with the concepts of "Order" and "Chaos", respectively. Energy determines the measure of possible useful work, the measure of the organization of the system. Therefore, it is logical to associate it with the concept of "Order". Entropy defines internal energy. It is logical to associate this with the measure of "Chaos". Through "Chaos", nature has found a way to combine two opposite concepts-movement and rest. "Chaos" ensures the existence of bodies at rest, if their elements are constantly in motion. This is possible because the total impulse of the elements can be equal to zero. Consequently, "Chaos" personifies the disappearance of motion or "Order" as a result of the disappearance of the energy of "Order", turning into the energy of "Chaos". Entropy is at its maximum when the system is in equilibrium. In this case, any subsystems allocated in it do not possess the energy of relative motion; do not possess the energy of "Order". Then the total energy of the system coincides with the internal energy of "Chaos" at zero energy of the "Order" of subsystems.

One of the key tasks of modern physics is to relate entropy with the dynamic parameters of the system, determined by the fundamental laws of classical mechanics (Zaslavsky, 1984; Lanczos, 1965). Without this, it is impossible to substantiate the empirical branches of physics, such as thermodynamics and kinetics, within the framework of the laws of classical mechanics.

Until recently, almost all existing concepts of entropy were either empirical, like the entropy of Clausius, or obtained within the framework of statistical laws, like, for example, the entropy of Boltzmann. The exception is the Kolmogorov-Sinai entropy (KS-entropy). It connects entropy with the exponential Lyapunov instability exponent for Hamiltonian systems (Zaslavsky, 1984).

It turned out that the physical meaning of the concept of entropy is well clarified in the framework of SB mechanics, where the concept of D-entropy is introduced. D-entropy connects the growth of the energy of "Chaos" due to the energy of its motion, that is, due to the energy of "Order". It is defined as the ratio of the increment of the body's internal energy to its total value. It should be emphasized that the internal energy in SB mechanics is the energy of the relative motion of the elements of the system. That is, the internal energy is associated 
with the dynamic, and not with the thermodynamic parameters of a body at rest, consisting of a large number of elements: temperature and pressure. D-entropy connects evolutionary changes in the structures of matter due to its interaction with the outside world. The concept of D-entropy is applicable for systems with any number of elements, since instead of the increment of heat, as in the case of the Clausius entropy, it takes the increment of internal energy, and instead of the temperature of the body, its total internal energy is taken (Somsikov, 2015). That is, D-entropy turns into Clausius entropy in the thermodynamic limit when the concept of body temperature is applicable. In this case, irreversibility is due to an increase in the D-entropy, when the energy of motion of the system of elements is converted into their energy of relative chaotic motion (Somsikov, 2021).

D-entropy follows directly from the equation of motion of the system. It is nonzero only in a non-uniform force field. Its nature is determined by the nonlinear interaction of micro- and macro-variables. The description of the dynamics of bodies using micro- and macro-variables was called a complete description of the dynamics of the system, since such a description allows one to construct the macro-dynamics of bodies based on the micro-dynamics of their elements. This also allows one to take into account the role of dissipative processes in the motion of bodies (Somsikov, 2015). Consequently, the D-entropy connects the violation of the symmetry of time with a change in the internal states of the system due to the energy of its motion. That is, D-entropy connects the concepts of "Chaos" and "Order" through the dynamic characteristics of systems. This means that deterministic chaos is a consequence of SB mechanics, and the laws of mechanics follow from the symmetry of three-dimensional space and the symmetry of time.

The mechanics of SB opened the possibility of substantiating thermodynamics. Justification is carried out based on a complete description of the dynamics of systems within the framework of the basic laws of physics. The use of D-entropy also makes it possible to substantiate statistical laws based on the equations of motion of SB. Since the D-entropy connects the motion of a body with its internal processes, it is also applicable to substantiate kinetics, since it allows one to determine the evolution of states of nonequilibrium bodies represented by a set of SBs obeying local thermodynamic equilibrium (Somsikov, 2010; Landau \& Lifshits, 1976). The limitations of the laws of statistics and the subordination of these laws to the fundamental laws of physics can be shown with an illustrative example. Let us imagine an equilibrium system of identical elements moving relative to each other at rest in a homogeneous space. The equilibrium of the system and the immobility of its center of mass mean that the sum of the momentums of its elements is equal to zero. On the other hand, this means the equiprobability of the directions of the momentum vectors of the elements of the system in space. Therefore, within the framework of probabilistic laws, there is always a nonzero probability that all elements will have a chosen direction. Then there is the possibility of movement of the system due to internal energy, which is forbidden for a homogeneous space according to Galileo's prin- 
ciple. From this, we see that we have the right to use the laws of probability only within the framework determined by the laws of physics. This strikes a blow to the holistic direction in philosophy (Inwagen \& Nozick, 2021; Levis, 1986). That is, according to the laws of mechanics, although the properties of the whole are qualitatively different from the properties of its parts. The property of the parts defines the property of the whole.

\section{The Interrelation of the Laws of the Steps of the Hierarchical Ladder of Matter}

Thus, the qualitative transition from the reversibility of the dynamics of structureless bodies to the irreversible dynamics of their systems follows from the fundamental laws of physics, using the principle of symmetry dualism. The fact that, according to the laws of physics, the behavior of systems follows from the laws of behavior of their elements significantly strengthens the position of reductionism and cognizability of natural evolutionary processes (Somsikov, 2010).

Taking into account the role of body structure in its dynamics led not only to the solution of the problem of irreversibility. He showed that, according to the laws of classical mechanics, matter is infinitely divisible and represents a hierarchy of open nonequilibrium dynamical systems (ONDS). That is, an element of both bone and living matter at all its hierarchical levels is ONDS (Somsikov, 2019; Somsikov, 2020). Without openness, the emergence, evolution and stationary states of ONDS, supported by external flows of matter or energy, are impossible. This conclusion is especially important from the point of view of solving the problem of a qualitative transition from the properties of bone to the properties of living matter.

When studying the dynamics of systems in an inhomogeneous force field, it was established that there are universal principles that determine the transition between the steps of the hierarchical ladder of matter, which can be represented by the ONDS hierarchy (Somsikov \& Azarenko, 2019). These principles, firstly, include the principle of interconnection of the laws of systems and their elements; the principle of interconnection of the parameters of the upper hierarchical levels of systems based on the parameters of the lower levels; the principle that the evolution of systems is the result of symmetry breaking.

Moreover, the symmetry breaking mechanism is universal for micro- and macro systems (Somsikov, 2019). That is, the ability to determine the laws of evolution of the upper hierarchical levels of ONDS based on the laws of dynamics of their lower hierarchical levels follows from the structural nature of matter. But if matter is the ONDS hierarchy, and if there are principles for constructing the laws of evolution of hierarchical structures of matter, based on knowledge of the laws of the dynamics of their elements, then knowing the laws that lie on one of the steps of the hierarchical ladder of matter, one can build a picture of the world by climbing the steps of the hierarchical stairs (Somsikov, 2021).

Thus, the results of the development of classical mechanics testify in favor of 
the idea of the existence of fundamental laws of physics and the possibility of constructing a deterministic picture of the world on their basis. But here the question of the possibility of constructing an evolutionary picture of the world on the basis of these fundamental laws, at least, is faced with the problem of infinite divisibility of matter. Optimism in overcoming this problem also inspires the possibility of the existence of universal principles that allow one to rise from the knowledge of matter at one of the steps of the hierarchical ladder to the knowledge of laws at the top step. It is clear that the infinity of the process of cognition of the diversity of all forms of matter follows from the condition of the infinity of the hierarchical ladder. But, at least in this case, there is no such form that sooner or later could not be cognized.

\section{The Unity of the Micro- and Macrocosm, the Principle of Causality}

The idea of the existence of fundamental laws of physics is based on the principles of causality. Nevertheless, this principle collides with the problems of the physics of the micro-world (Tan, 2020). The nature of these problems is primarily related to the Geizenberg uncertainty principle, according to which it is impossible to accurately determine the states of microparticles. Consequently, in the microcosm the present is probabilistically connected with the past (Geizenberg, 1968, 1989; Werner \& Farrelly, 2019; Shirazi, 2020). This violates the principle of causality, without which it is impossible to build an evolutionary picture of the world. However, relying on the mechanics of SB, there is a possibility of a deterministic interpretation of the Heisenberg uncertainty principle, without going beyond the framework of deterministic laws. Let us substantiate this statement.

If matter is infinitely divisible, then it must be the ONDS hierarchy. That is, a microparticle, no matter how small it is, must have a structure and internal energy. But in this case, the principles of forming macro-systems from micro-systems are correct. Then the use of the canonical Schrödinger equation will lead to uncertainty of their trajectory. Indeed, this equation was obtained based on the canonical formalism of classical mechanics, which does not take into account the role of the structure of the system in its dynamics. In this case, the uncertainty of the state of a dynamic quantum system is analogous to the uncertainty of the trajectory that arises when describing the dynamics of a classical system using Newton's motion equation, which does not take into account the structure of the body. As in classical mechanics, this uncertainty will be determined by the relative proportion of changes in the internal energy to the energy of motion during the interaction of systems. In the simplest case, an oscillator can represent ONDS. In accordance with the laws of quantum mechanics, the internal energy, its change during the interactions of the oscillators cannot be equal to zero. This is consistent with the mechanics of SB. It is obvious that considering this circumstance will undoubtedly give uncertainty in calculating the 
phase volume of interacting quantum systems. This uncertainty is comparable to the value of Planck's constant. Thus, the probabilistic nature of the dynamics of quantum particles, which follows from the canonical Schrödinger equation, may be due to the lack of taking into account the influence of the structure of quantum particles on their dynamics. This uncertainty can be eliminated if the extended Schrödinger equation is used to describe the dynamics of quantum particles, which takes into account the role of the D-entropy in their dynamics (Somsikov, 2017).

Thus, proceeding from the physics of evolution, the uncertainties of the dynamics of quantum particles are associated with the limitations of the methods of their description, but not with the nature of the micro-world. This conclusion is confirmed by a numerical experiment revealing the passage of a classical oscillator through a potential barrier, the height of which is greater than the energy of motion of the oscillator, but less than the total energy. This effect occurs only when the role of the D-entropy in the dynamics of systems is taken into account.

There is also one more mechanism of uncertainty (Landau \& Lifshits, 1989). Indeed, the measurements themselves are always associated with the impact on the system. For quantum particles, the energy of this effect, for example, by a quantum of light, can be commensurate with the energies of their motion, which will introduce uncertainty in the measurement results.

Thus, if we take into account the infinite divisibility of matter and its structure, then the problem of the existence of determinism in the quantum world can disappear, which testifies in favor of the unity of the laws of physics and the existence of fundamental laws of physics. Here, however, many open questions remain, in particular, how to interpret the concept of quantum-wave dualism, how to interpret quantum entanglement, how to connect quantum mechanics with the field form of matter, etc. (Geizenberg, 1989; Belinsky, 2003). These pending questions should also be considered from the point of view of the structure of matter.

\section{Evolution Physics and Reality}

One of the key elements of the evolutionary picture of the world is the Universe. In addition to the fact that its study is independent, it is convenient because it allows us to observe objects in the Universe, for example, stars, simultaneously at all stages of their evolution. That is, it is possible to simultaneously study the laws of evolution at all stages of the formation and decay of systems. But the study of the physics of evolutionary processes of matter until recently faced with problems of explaining the second law of thermodynamics. Therefore, I. Prigogine called modern physics "the physics of existence", since it did not explain the processes of evolution (Nicolis \& Prigogine, 1990). So, today astrophysics relies on the Einstein equation. This is a relativistic analogue of Newton's equation of motion for a structureless MP. But Newton's equation and the formalisms built on its basis can be used only in a limited framework to study astrophysical ob- 
jects, since they do not describe evolutionary processes. The question arises: how justified are attempts to build a model of the Universe based on equations that do not allow describing irreversible evolutionary processes? After all, it is possible that it is precisely these shortcomings and the contradictions arising in connection with them between the results of observations of the Universe and theories that largely force us to put forward hypotheses about hidden matter, dark energy, etc. But the physics of evolution gives hope that many of these contradictions will be resolved. For example, a change in the internal energy of a star during its motion in inhomogeneous gravitational fields, described in the framework of the physics of evolution, will amend the energy balance of stars. Therefore, for example, stars can emit more energy than the estimates of their internal sources give without taking into account the possibility of increasing their internal energy due to inhomogeneities of the external force fields. Consequently, taking into account the role of the structure of cosmological objects in their motion will lead to corrections. It is possible that these amendments will significantly change our understanding of the physics of space (Milgrom, 1983).

From the physics of ONDS, it follows that for the formation of new objects in the Universe, it must expand. This will provide it with negentropy, which compensates for the growth of D-entropy and will lead to the organization of new structures, which corresponds to the conclusions based on the thermodynamics of the Universe (Tolman, 1974; Rumer \& Ryvkin, 1977).

The Earth's atmosphere, including ionospheric plasma, is an illustrative example and a very convenient object for studying the regularities of ONDS behavior. For example, it is convenient to study how the gradients of external forces create the internal heterogeneous structures of the atmosphere. The global source of such inhomogeneities is the solar terminator-the area of solar radiation flux gradients in the Earth's atmosphere caused by the sphericity of the Earth and the Sun (Somsikov \& Troitsky, 1975; Somsikov, 1983).

Note that plasma is a convenient object for studying the evolutionary processes of structure formation. For example, the so-called "plasma crystal" was first discovered experimentally in dusty plasma. The formation time of such a plasma crystal is rather short, which makes plasma a convenient object for studying the processes of structure formation (Ramazanov et al., 2008; Baimbetov \& Ramazanov, 1998). The study of such systems will contribute to the development of the physics of evolution, which includes the mechanics of SB, ONDS, extended formalisms of classical mechanics, principles of interrelation of hierarchical levels for ONDS, obtained based on the SB equations of motion.

It is obvious that the replacement of a MP by an ONDS, consisting of a set of SBs, does not exhaust all the possibilities for the further development of the physics of evolution. Indeed, according to the laws of classical mechanics, matter is infinitely divisible. But in this case, the ONDS, built from the MP, although it allows us to understand the basic laws of the evolution of matter, is nevertheless an approximation to reality. Therefore, in the future, it will be necessary to take 
into account the infinite divisibility of matter in the equations of dynamics. This requirement can be met if we accept the condition that matter has a field form (Alexeyeva, 2009). Perhaps there is a way to explain the relationship between the nature of matter and field.

\section{Conclusion}

The development of an evolutionary picture of the world is faced with the problem of creating and developing theories capable of describing the evolutionary processes of nature. Expanding the scope of classical mechanics, achieved by replacing the body model in the form of an MP in the equation of motion with a body model in the form of ONDS and using the principle of symmetry dualism, largely solves this problem. As a result of this expansion, it was established that the infinite divisibility of matter and the hierarchical structure of matter from simple to complex follow the fundamental laws of physics. Also, universal principles have been established that make it possible to determine the laws of behavior of the upper hierarchical level of matter on the basis of the laws of its behavior at the lower level, which significantly expands the possibilities of constructing a picture of the world.

Within the framework of the obtained extension of mechanics, it became possible to substantiate thermodynamics, statistical physics, kinetics based on the laws of classical mechanics. It was possible to show that Heisenberg's uncertainty can be associated with the lack of the necessary consideration of the role of the structure of quantum particles in their interactions when using the existing formalisms of quantum mechanics. Consequently, such accounting can lead to the elimination of the uncertainty principle. This will not only expand the possibilities of constructing an evolutionary picture of the world, but will also strengthen the position of determinism.

The existence of fundamental laws of physics and universal principles that determine the relationship between the laws of behavior of matter at adjacent steps of its hierarchical ladder opens up the possibility of establishing a relationship between bone and living matter (Nelson \& Cox, 2017; Levis, 1986). This allows you to build a holistic picture of the world, climbing the steps of the hierarchical structure of matter from simple to complex, eliminating the problems of inconsistency of existing areas of natural science. Overall, this testifies to the validity of the ideas of universal evolutionism (Knyazeva \& Kurdyumov, 2005; Loskutov \& Mikhailov, 1990).

\section{Acknowledgements}

The work was carried out with the financial support of the Committee of Science of the Ministry of Education and Science of the Republic of Kazakhstan grant project AP09259554.

\section{Conflicts of Interest}

The authors declare no conflicts of interest regarding the publication of this paper. 


\section{References}

Alexeyeva, L. A. (2009). Newton's Laws for a Biquaternionic Model of Electro-Gravimagnetic Fields, Charges, Currents, and Their Interactions. Journal of Physical Mathematics, 1, Article ID: S090604. https://doi.org/10.4303/jpm/S090604

Baimbetov, N. F., \& Ramazanov, T. S. (1998). On the Formation of Ordered Structures in a Nonideal Plasma. FNTP, 1, 490-500.

Baumgarten, C. (2017). The Final Theory of Physics-A Tautology? arXiv:1702.00301v1 [physics.gen-ph] 25 Jan 2017.

Belinsky, A. V. (2003). Quantum Nonlocality and the Absence of a Priori Values of Measured Quantities in Experiments with Photons. Physics-Uspekhi, 46, 877. https://doi.org/10.1070/PU2003v046n08ABEH001393

Geizenberg, V. (1968). Planck's Opening and the Basic Philosophical Problems of the Nuclear Theory. UFN, 2, 163-175.

Geizenberg, V. (1989). Physics and Philosophy. A Part and Whole. Moscow: Sci. Press.

Goldstein, G. (1975). Classical Mechanics. Moscow: Sci. Press.

Hooft, G. W. (2017). Free Will in the Theory of Everything. arXiv:1709.02874v1 [quant-ph] 8 Sep.

Inwagen, P., \& Nozick, R., (2021). The Probabilistic Argument for Why Anything Exists at All Examined.

https://thinkingdeeply.medium.com/the-probabilistic-argument-for-why-anything-exi sts-at-all-examined-van-inwagen-nozick-6fd7f209d11d

Knyazeva, E. N., \& Kurdyumov, S. P. (2005). Foundations of Synergetic. Synergetic Worldview. Moscow: Kom Kniga.

Lanczos, K. (1965). Variational Principles of Mechanics. Moscow: Mir.

Landau, L. D., \& Lifshits, E. M. (1973). Mechanics. Moscow: Sci. Press.

Landau, L. D., \& Lifshits, E. M. (1976). Statistical Physics. Moscow: Sci. Press. https://doi.org/10.1007/978-3-642-97294-2

Landau, L. D., \& Lifshits, E. M. (1989). Quantum Mechanics. Moscow: Sci. Press.

Levis, D. (1986). On the Plurality of Worlds. Oxford: Basil, Blackwell.

Loskutov, Mikhailov A.Yu., A.S. (1990). Introduction to Synergetics. Moscow: Sci. Press.

Mac Voy, K. (1967). Symmetry Groups in Physics. UFN, 91, 121-150.

Milgrom, M. A. (1983). Modification of the Newtonian Dynamics as a Possible Alternative to the Hidden Mass Hypothesis. Astrophysics Journal, 1, 365-370.

https://doi.org/10.1086/161130

Nelson, D. L., \& Cox, M. M. (2017). Lehninger Principles of Biochemistry. Amazon.Com.

Nicolis, G., \& Prigogine, I. (1990). Exploring Complexity. Moscow: Mir Press.

Ramazanov, T. S., Dzhumagulova, K. N., Jumabekov A. N., \& Dosbolayev M. K. (2008). Structural Properties of Dusty Plasma in Direct Current and Radio Frequency Gas Discharges. Physics of Plasmas, 15, 053704. https://doi.org/10.1063/1.2918336

Rumer, Yu., \& Ryvkin, M. S. (1977). Thermodynamics Stat. Physics and Kinetics. Moscow: Sci. Press.

Shirazi, A. N. (2020). Heisenberg's Equality of in Equivalents Problem. arXiv:2003.06517v1 [physics.hist-ph]

Somsikov, V. M. (1983). Solar Terminator and Atmospheric Dynamics. Flma-Ata. Nauka: Alma-Ata. Sci. Press. 
Somsikov, V. M. (2007). Problems of Evolution of Open Systems. PEOS, 9, 5-16.

Somsikov, V. M. (2010). On the Principles of Constructing the Mechanics of Structured Particles Based on the Mechanics of a Material Point. PEOS, 2, 3-17.

Somsikov, V. M. (2015). The Dynamical Entropy. International Journal of Sciences, 4, 30-36. https://doi.org/10.18483/ijSci.712

Somsikov, V. M. (2016). Transition from the Mechanics of Material Points to the Mechanics of Structured Particles. Modern Physics Letters B, 4, 1-11.

Somsikov, V. M. (2019). The Evolution and Breaking Symmetry in the Physics. In 13th Chaotic Modeling and Simulation International Conference (pp. 777-787). Florence: Springer.

Somsikov, V. M. (2020). Deterministic Irreversibility Mechanism and Basic Element of Matter. In C. Skiadas, \& Y. Dimotikalis (Eds.), 12th Chaotic Modeling and Simulation International Conference, Springer Proceedings in Complexity (pp. 245-256). Cham: Springer. https://doi.org/10.1007/978-3-030-39515-5 20

Somsikov, V. M. (2021). Foundations of the Physics of Evolution. Almaty: KazNU.

Somsikov, V. M., \& Azarenko, S. N. (2019). Determinism in Physics and Cognoscibility of a Picture of the World. Open Journal of Philosophy, 9, 265-280.

https://doi.org/10.4236/ojpp.2019.93018

Somsikov, V. M., \& Troitsky, B. V. (1975). Generation of Disturbances in the Atmosphere When the Solar Terminator Passes through It. Geomagnetism and Aeron, 15, 856-860.

Somsikov, V. M. (2017). Extension of the Schrodinger Equation. EPJ Web of Conferences, 138, Article ID: 07003. https://doi.org/10.1051/epjconf/201713807003

Tan, W. P. (2020). No Single Unification Theory of Everything. arXiv:2003.04687v1 [physics.gen-ph] 7 Mar 2020.

Tolman, R. (1974). Relativity, Thermodynamics and Cosmology. Moscow: Sci. Press.

Weinberg, S. (1992). Dreams of a Final Theory. New York: Vintage.

Werner, R. F., \& Farrelly, T. (2019). Uncertainty from Heisenberg to Today. Foundations of Physics, 49, 460-491. https://doi.org/10.1007/s10701-019-00265-Z

Zaslavsky, G. M. (1984). Stochasticity of Dynamic Systems. Moscow: Sci. Press. 